Contents:

DNA sequences

Preparation and gel characterisation of U-shaped motifs

Preparation of single-stranded scaffold for T-motif origami structures

Atomic Force Microscopy (AFM) of T-motif origami structures

Simulation Methods

Additional Simulation Results 


\section{DNA sequences}

DNA oligonucleotides were purchased from Integrated DNA Technologies, Inc. (IDT); unpurified except for those with fluorescent labels (HPLC purified) and those over 60 bases in length (PAGE purified). Strands were resuspended at $100 \mu \mathrm{M}$ (or $20 \mathrm{ng} / \mu \mathrm{L}$ for the scaffold strand of the T-motif origami motifs) in TE. All were provided single-stranded except the scaffold strand. DNA sequences (loops and sticky-ends which form T-motifs are in red and green respectively):

\section{U-shaped motif (Figure 1)}

Five strands form the double-stranded template:

1. TTGGCCATGGTAGTCCCAGCGAACGGAGAACCTGCCGCGCCGACAAGGCCGGTCCCTGTCGGCGAATCGCGCCG

2. TGGGACTAGTGTCCCCATGGCCAA

3. (5' TAMRA) CGGCAGGTTCTGTCGTCGCCGTTCGC

4. GGACCGGCGCTGGCTTGTCGGCG

5. CGGCGCGATTCCACTCGGCCGACAG

Linkers:

Double-stranded linkers are formed by hybridisation of the short strand (GCCAGGTGTGCCAGAGCAGG) to strands $L_{00}, L_{01}, L_{10}$ or $L_{11}$.

Loo: CGTGTACCCTGCTCTGGCACACCTGGCCGCTG

L01: CGTGTACCCTGCTCTGGCACACCTGGCCGAGTG

L10: GACACCCTGCTCTGGCACACCTGGCCGCTG

L11: GGACACCCTGCTCTGGCACACCTGGCCGAGTG

T-motif origami motifs (Figures 2, 3 and 4)

Scaffold (537 nt):

CTGCGTAAGCTTCGTATTAAATCCTTTGCCCGAACGTTATTAATTTTAAAAGTTTGAGTAACATTATCATTTTGCGGAACAAAGAAACCACCAGAAGG AGCGGAATTATCATCATATTCCTGATTATCAGATGATGGCAATTCATCAATATAATCCTGATTGTTTGGATTATACTTCTGAATAATGGAAGGGTTAG AACCTACCATATCAAAATTATTTGCACGTAAAACAGAAATAAAGAAATTGCGTAGATTTTCAGGTTTAACGTCAGATGAATATACAGTAACAGTACCT TTTACATCGGAGGAAACAATAACGGATTCGCCTGATTGCTTTGAATACCAAGTTACAAAATCGCGCAGAGGCGAATTATTCATTTCAATTACCTGAGC AAAAGAAGATGATGAAACAAACATCAAGAAAACAAAATTAATTACATTTAACAATTTCATTTGAATTACCTTTTTTAATGGAAACAGTACATAAATCA ATATATGTGAGTGAATAACCTTGCTTCTGTAAATCGTCGACTGGGTA

Loop strands:

1. TACCCAGTCGACGATtTACAGAgGCTCAGCAAGGT

2. TATTCACTGTGTCGCACATATATTG

3. АTTTATGTACCGACGTGTTTCCA

4. TTAAAAAACAGGTGGGTAATTCAAA

5. TGAAATTGTTGGCTCAAATGTAA

6. TTAATTTTGTGTCGGTTTTCTTGAT

7. GTTTGTTTCACGACGTCATCTTC

8. TTTTGCTCCAGGTGAGGTAATTGAA

9. ATGAATAATTGGCTCCGCCTCTG

10. CGCGATTTGTGTCGTGTAACTTGGT

11. ATTCAAAGCACGACGATCAGGCG

12. AATCCGTTCAGGTGATTGTTTCTCC

13. CGATGTAAAAGGCTCGGTACTGT

14. TACTGTATGTGTCGATTCATCTGAC
15. GTTAAACCTGCGACGAAAATCTA

16. CGCAATTTCAGGTGCTTTATTTCTG

17. TTTTACGTGCGGCTCAAATAATT

18. TTGATATGGTGTCGGTAGGTTCTAA

19. СССTTCCATTCGACGATTCAGAA

20. GTATAATCCAGGTGCAAACAATCAG

21. GATTATATTGGGCTCATGAATTG

22. CCATCATCGTGTCGTGATAATCAGG

23. AATATGATGACGACGTAATTCCG

24. СTССTTCTCAGGTGGGTGGTTTCTT

25. TGTTCCGCAAGGCTCAATGATAA

26. TGTTACTCGTGTCGAAACTTTTAAA

27. ATTAATAACGCGACGTTCGGGCA

28. AAGGATTTCAGGTGAATACGAAGCTTACGCAG

splint (replaces 14 and 15 in compact motif) TACTGTATATTCATCTGACGTTAAACCTGAAAATCTA 
Loop strands with complementary tails (in blue; Figure $2 \mathrm{~d}$ and e):

1 + 8-nt complementary tail: GTTCAGCGTAGAGTCGACGATTTACAGAGGCTCAGCAAGGT

28 + 8-nt complementary tail: AAGGATTTCAGGTGAATACGAAGCTTGGCGTAATCGCTGAAC

1 + 10-nt complementary tail: GTTCAGCGCGTAGAGTCGACGATTTACAGAGGCTCAGCAAGGT

28 + 10-nt complementary tail: AAGGATTTCAGGTGAATACGAAGCTTGGCGTAATCGCGCTGAAC

Linkers:

Double-stranded linkers are formed by hybridisation of the linker strand (GCCAGGTGTGCCAGAGCAGG) to strands $L_{a, b}, L_{c, d}$ or $L_{d, c}$.

$L_{a, b}$ : САССтGстястстGGCACACCTGGCGAGCC

$L_{c, d}:$ CGACACCCTGCTCTGGCACACCTGGCCGTCG

Ld,c: CGTCGCCTGCTCTGGCACACCTGGCCGACAC

\section{Preparation and gel characterisation of U-shaped motifs}

Template and linker strands were annealed $\left(95\right.$ to $20^{\circ} \mathrm{C}$ at a rate of $\left.-1^{\circ} \mathrm{C} / \mathrm{min}\right)$ together in TAE/Mg ${ }^{2+}$ buffer $(40 \mathrm{mM}$ Tris, $20 \mathrm{mM}$ acetic acid, $1 \mathrm{mM}$ EDTA, $12.5 \mathrm{mM} \mathrm{MgCl}_{2}$, pH8.5). All strands were at $150 \mathrm{nM}$ except the labelled template strand at $100 \mathrm{nM}$. Polyacrylamide gel electrophoresis (PAGE) was carried out using 8\% 29:1 acrylamide in TAE/Mg ${ }^{2+}$ and cast using the Bio-Rad Mini-Protean casting stand, clamps, plates with $0.75 \mathrm{~mm}$ spacer, and 15 -well comb. $8 \mu \mathrm{L}$ of sample $+2 \mu \mathrm{L}$ of $5 \mathrm{x}$ loading buffer was used per well. Gels were run using the Mini-Protean Tetra Cell at $150 \mathrm{~V}$ and $4^{\circ} \mathrm{C}$ for 90 minutes, using TAE/Mg ${ }^{2+}$ as the running buffer. Gels were imaged using a Pharos FX Plus Molecular Imager.

\section{Preparation of single-stranded scaffold for T-motif origami structures}

The scaffold strand was provided by IDT as a double-stranded gBlock gene fragment, which was amplified and made single-stranded by PCR using a 5'-OH forward primer (CTGCGTAAGCTTCGTATTAAATCC) and a phosphorylated reverse primer (5' PTACCCAGTCGACGATTTACAGAAG), followed by lambda exonuclease digestion of the 5 ' phosphate strands. $100 \times 50 \mu \mathrm{L}$ reactions were prepared using Q5 High-Fidelity 2x Master Mix (New England Biolabs; NEB) and subjected to the thermocycling conditions recommended by NEB (annealing temperature was $65^{\circ} \mathrm{C}$ and 30 cycles were carried out). Samples were pooled and purified using the QIAquick PCR Purification Kit (Qiagen). This produced $600 \mu \mathrm{L}$ of sample which was incubated at $37^{\circ} \mathrm{C}$ for 45 minutes with $160 \mu \mathrm{L}$ lambda exonuclease (5000 U/mL; NEB), $100 \mu \mathrm{L} 10 \mathrm{x}$ lambda exonuclease buffer and $140 \mu \mathrm{L}$ Milli-Q water, before heat inactivation of the enzyme at $75^{\circ} \mathrm{C}$ for 15 minutes. This sample was again purified using the QIAquick PCR Purification Kit.

\section{Preparation and gel characterisation of T-motif origami structures}

Single-stranded scaffold (at $200 \mathrm{nM}$ ) and loop/splint strands (at $300 \mathrm{nM}$ ) were annealed together in TAE/Mg ${ }^{2+}$ buffer to form the Tmotif origami template. Linker strands (at $5 \mu \mathrm{M}$ ) were either annealed with the template or incubated with the annealed template at room temperature for 1 hour. Agarose gel electrophoresis was carried out using $0.7 \%$ agarose in TAE buffer, cast using the Bio-Rad Sub-Cell GT Tray $(15 \times 10 \mathrm{~cm})$ with a 20 -well comb. $8 \mu \mathrm{L}$ of sample $+2 \mu \mathrm{L}$ of $5 x$ loading buffer was used per well. Gels were run using the Mini-Protean Tetra Cell at $60 \mathrm{~V}$ and $4^{\circ} \mathrm{C}$ for 2 hours, using TAE/ $\mathrm{Mg}^{2+}$ as the running buffer. Gels were imaged using an Amersham Typhoon Imager.

\section{Atomic Force Microscopy (AFM) of T-motif origami structures}

Samples prepared as above were gel purified by cutting out gel bands and squeezing to remove the liquid. Samples were diluted to a template concentration of $20 \mathrm{nM}$ in filtered imaging buffer (1x TAE (40 mM Tris, $20 \mathrm{mM}$ acetic acid, $1 \mathrm{mM}$ EDTA-Na $), 12.5 \mathrm{mM}$ magnesium acetate, $4 \mathrm{mM} \mathrm{NiCl}_{2} \mathrm{pH} 8.5$ ). AFM images were acquired using a Cypher ES AFM (Oxford Instruments) in tapping mode in liquid using the blueDrive Photothermal Excitation option (with the $0.01 \mathrm{x}$ filter cube) and AC40 cantilevers (Bruker). $5 \mu \mathrm{L}$ of diluted sample was pipetted onto freshly cleaved mica, left to adhere, and then covered with $60 \mu \mathrm{L}$ imaging buffer. The tip was also covered with $50 \mu \mathrm{L}$ of imaging buffer before beginning image acquisition. 


\section{Simulation Methods}

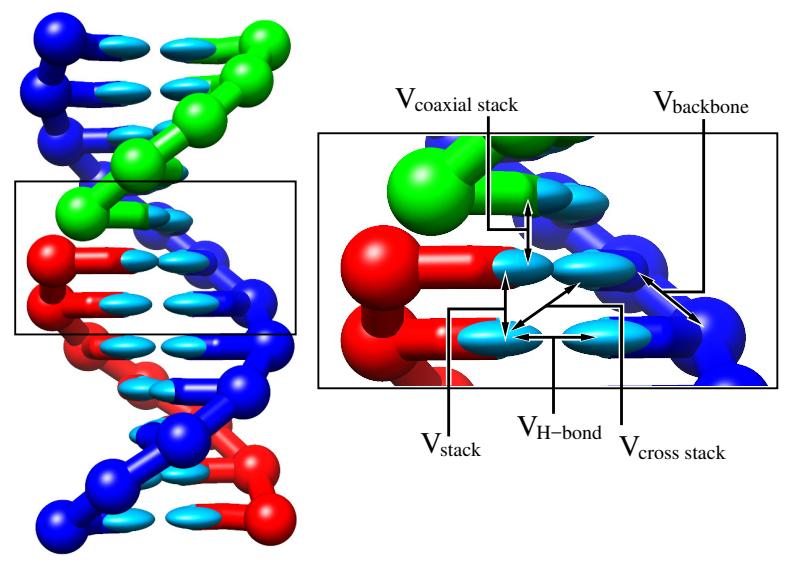

Figure S1. Simplified representation of a 12 base-pair double helix, illustrating the various interactions in the oxDNA model. Each nucleotide is taken as a rigid body, interacting with other nucleotides through three interaction sites: a backbone connectivity site, a hydrogen boding site and a stacking site. The interaction potential for adjacent nucleotides on a strand contains backbone connectivity and stacking terms. All other pairs of nucleotides interact through hydrogen bonding, cross stacking and coaxial stacking potentials. In addition, excluded volume terms are included in each pair-wise interaction potential and effective electrostatic interactions are treated in a mean-field approach based on the Debye-Huckel model for screened electrostatics. For a detailed description of the oxDNA potential, see reference 31 of the accompanying manuscript.

To calculate the free energies shown in Figure 1c, we simulate our systems using the Virtual Move Monte Carlo (VMMC) of Whitelam et al. ${ }^{1} \mathrm{VMMC}$ is a cluster move algorithm that allows collective movement of strongly interacting particles to sample efficiently conformations of strands from the canonical ensemble. In addition, we use the umbrella sampling technique ${ }^{2}$ to speed up sampling of transitions between metastable states. To achieve this, interaction potentials are artificially biased to flatten energy barriers over order parameters (observables) of interest. In this work, we break up our simulations into two umbrella sampling windows for hybridisation of each sticky end to its corresponding bulge loop. In the first window, the minimum end-to-end distance between centre of mass of nucleotides of the bulge loop and those of the sticky end is taken as the biased order parameter. In the second window, we apply the bias to the number of base-pairs between the bulge loop and the sticky end. Two nucleotides are considered basepaired if the hydrogen boding interaction strength between them is greater than 0.093 times the well-depth of the potential (approximately $0.6 \mathrm{kcal} \mathrm{mol}^{-1}$ at $25^{\circ} \mathrm{C}$ ). Since the applied biasing weights are known, the resulting probability distributions can then be easily unbiased for each simulation window. Finally, we use the Weighted Histogram Analysis Method ${ }^{3}$ to patch up probability distributions from individual umbrella sampling windows and construct complete free-energy profiles.

To simulate the T-junction origami using oxDNA we use molecular dynamics with an Andersen-like thermostat. ${ }^{4}$ To apply tensile forces to the origami, we follow the approach used in Ref. 5. Specifically, we apply harmonic traps to the end nucleotides, with the displacements of these nucleotides from the centres of the traps allowing the forces exerted by the traps to be measured. The traps are moved in opposite directions at a constant rate. The force constant of the traps were $5.71 \mathrm{pN} / \mathrm{nm}$, and we considered three different trap velocities, namely $2.81 \times 10^{4}, 2.81 \times 10^{5}$ and $2.81 \times 10^{6} \mathrm{~nm} / \mathrm{s}$. These correspond to force loading rates of $3.21 \times 10^{6}$, $3.21 \times 10^{7}, 3.21 \times 10^{8} \mathrm{pN} / \mathrm{s}$. The forces reported in the figures are for the components of the force in the direction of trap motion averaged over both ends. To reduce the fluctuations in the force-extension curves associated with oscillations in the trap, the reported forces were also averaged over 10 data points. The simulations were run at $25^{\circ} \mathrm{C}$ and a $\left[\mathrm{Na}^{+}\right]$concentration of $1 \mathrm{M}$.

\section{Additional simulation results}

Movies showing simulation trajectories for the two origami in solution are included as supplementary material. These illustrate that, although the origamis have a well-defined structure as designed, they also possess significant flexibility.

In Figure S2 we show the force-extension curves for the two motifs at the three different force loading rates that we used. Movies of the trajectories for these six pulling simulations are included as Supplementary Material. The decreases in the rupture forces as the force loading rates decrease are significant.

For the compact motif, the sawtooth character of the curves is apparent for all three loading rates, with the drops being associated with the dissociation of one end of a single linker, or occasional two consecutive dissociation events. The pathway for all three runs is very similar with the long-range links breaking first. Also apparent is the similarity between trajectories that traverse the same series of intermediate states. For example, for the compact trajectories shown the transitions between the first three intermediates occur at lower forces and extensions for lower pulling rates but are recognisably the same. This feature also allows one to quickly see when the different trajectories follow a slightly different pathway. For example, the pathway for the intermediate loading rate is slightly different in the middle than for the slowest loading rate, because one of the short-range links happens to break before all the longrange links have broken (Figure S3).

For the extended motif the rupture forces are always higher than for the compact motif at the equivalent loading rate. This is because the tensile force is shared between the two long edges of the ladder-like motif, rather than concentrated in a single linker. The force is less effectively shared between the two edges at either end of the ladder, and it is at the ends of the ladder that linkers are more 
likely to dissociate (Figure S4 illustrates one such event). As all the linkers mediate local connections, the force drops are relatively small and become better resolved at slower loading rates. The linkers are also much more likely to dissociate from the less-stable 3' ends; at the slowest loading rate this is the case for all 13 linkers. By contrast, for the compact motif, there is tendency for the pairs of long-range linkers to dissociate from the same side, presumably because of an asymmetry due to the presence of the partly bound linker, leading these linkers to remain attached by a mixture of $3^{\prime}$ and $5^{\prime}$ ends.
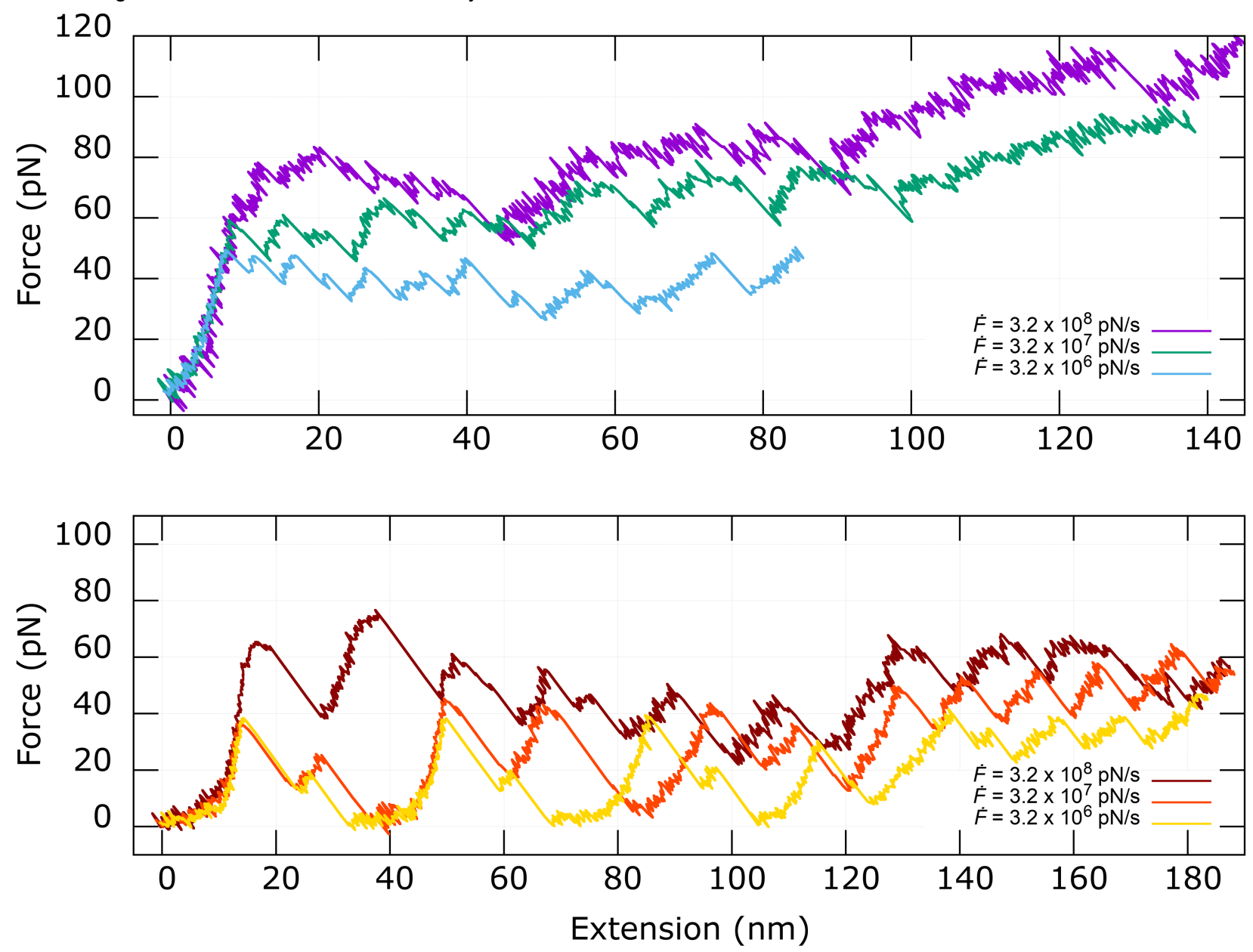

Figure S2. Force-extension curves for the extended motif (top) and the compact motif (bottom) for three different force loading rates, as labelled. 


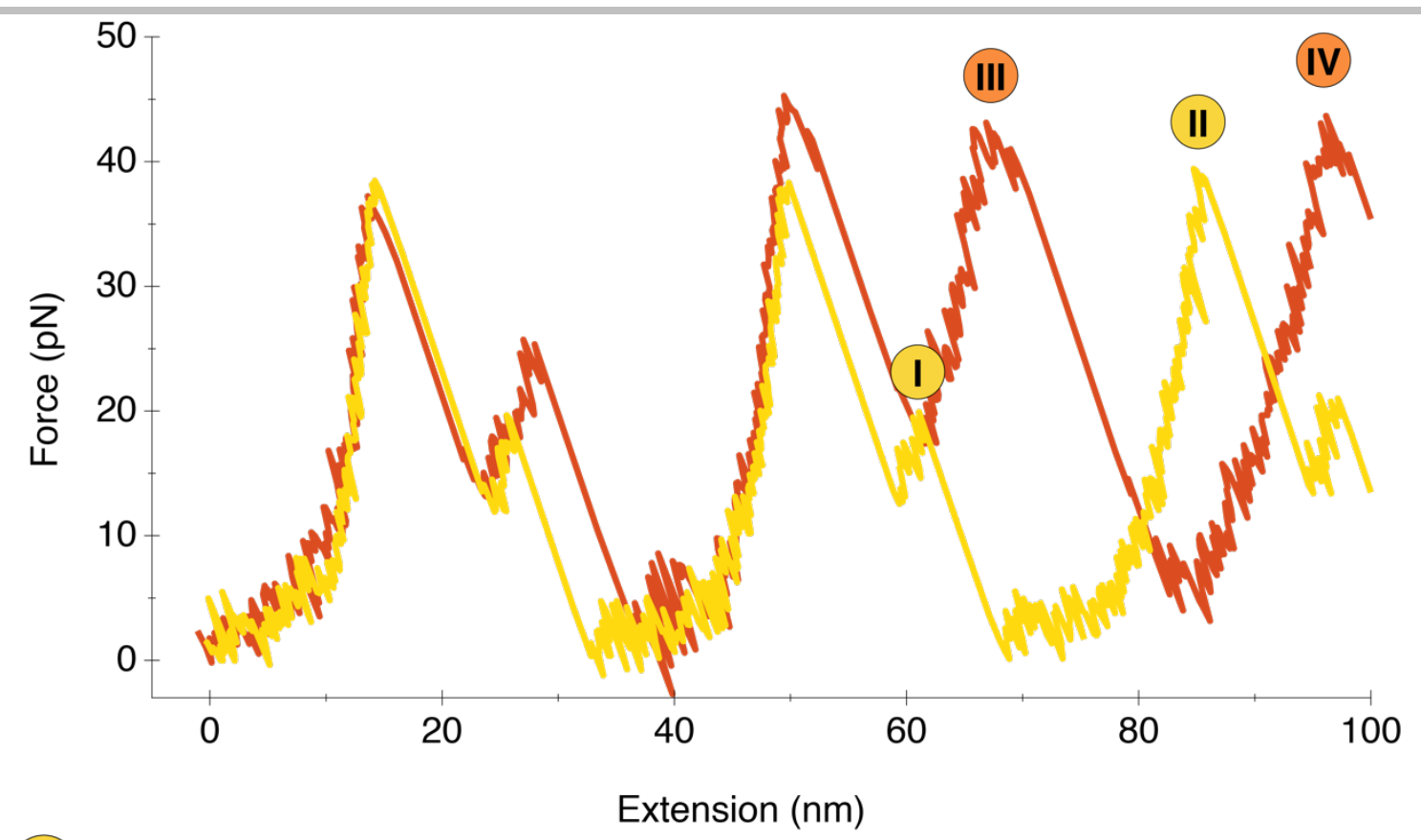

(I)

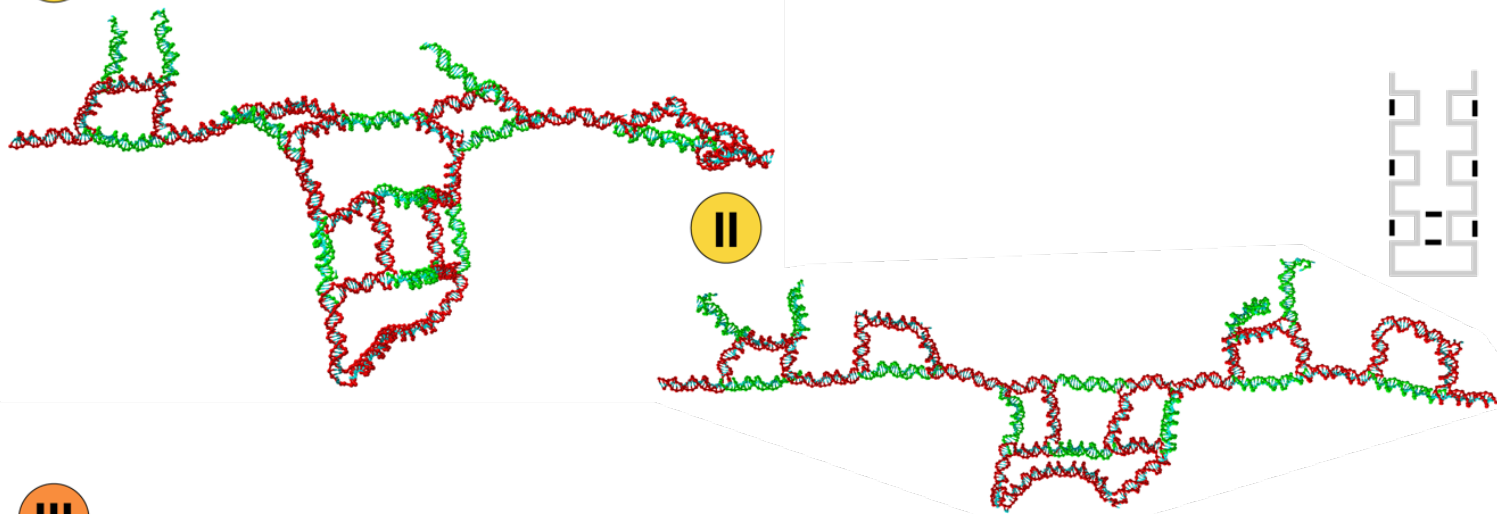

(III)

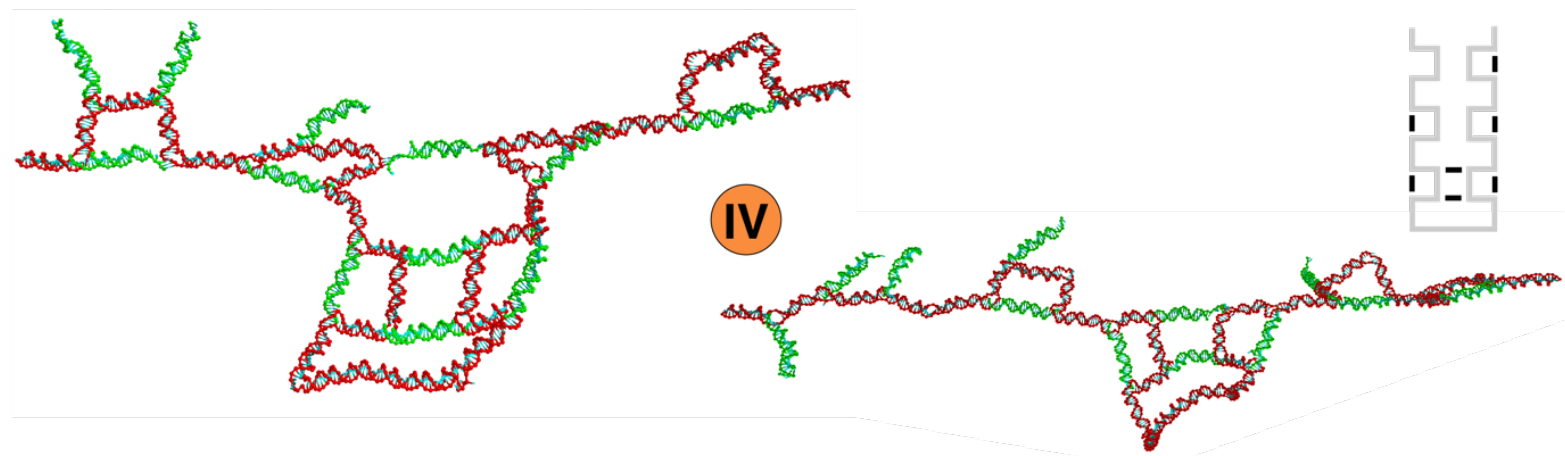

Figure S3. Different trajectories follow slightly different pathways. A short-range interaction breaks early in the pathway at the intermediate loading rate (orange curve) compared with the pathway at the slowest loading rate (yellow curve). 

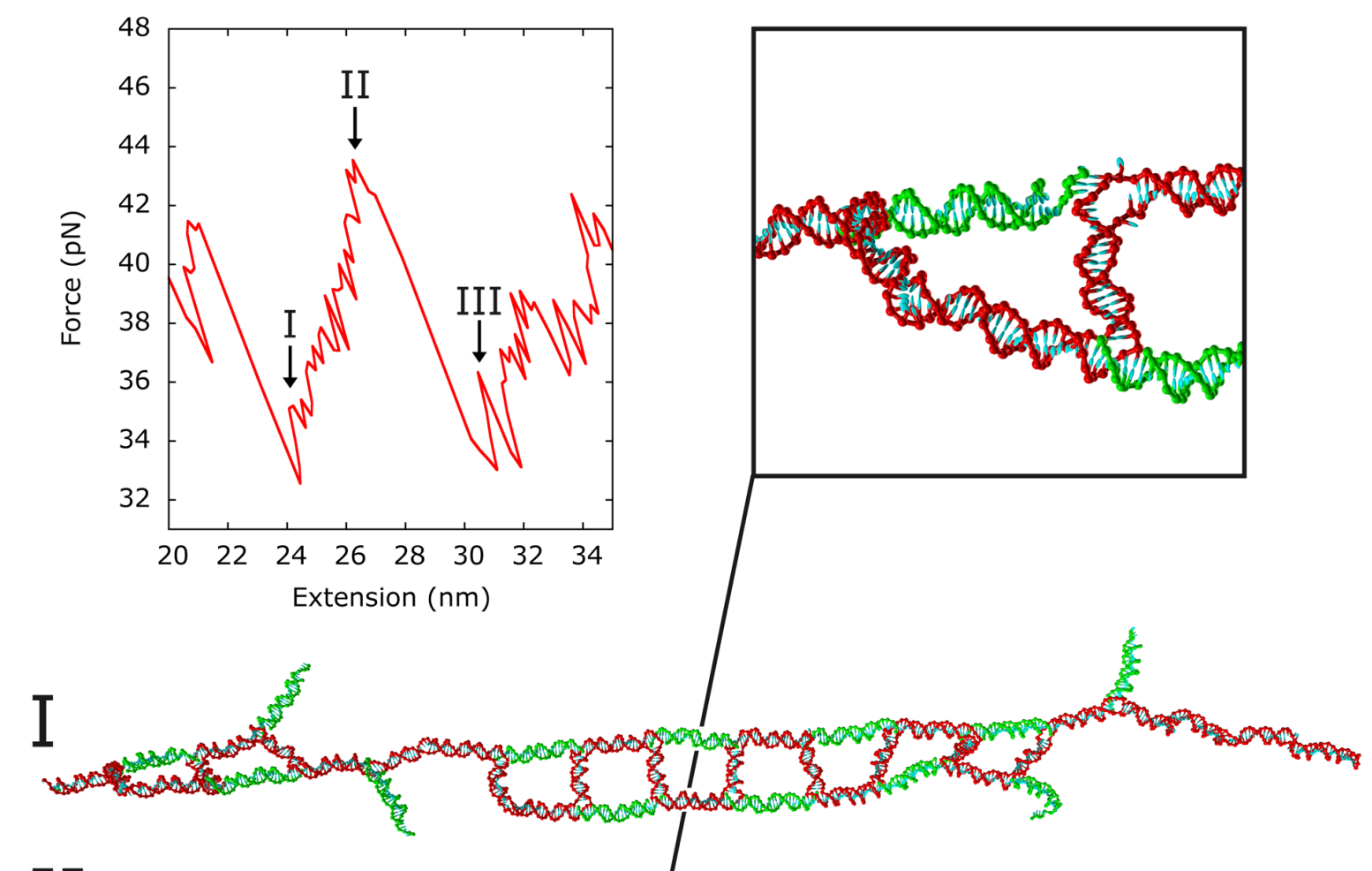

\section{II}

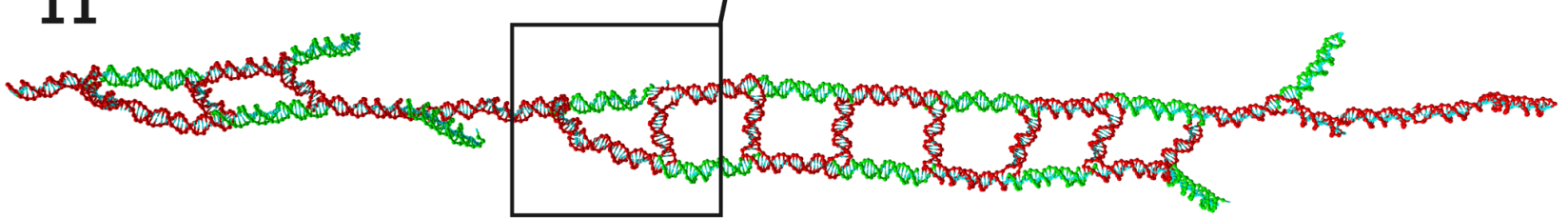

\section{III}

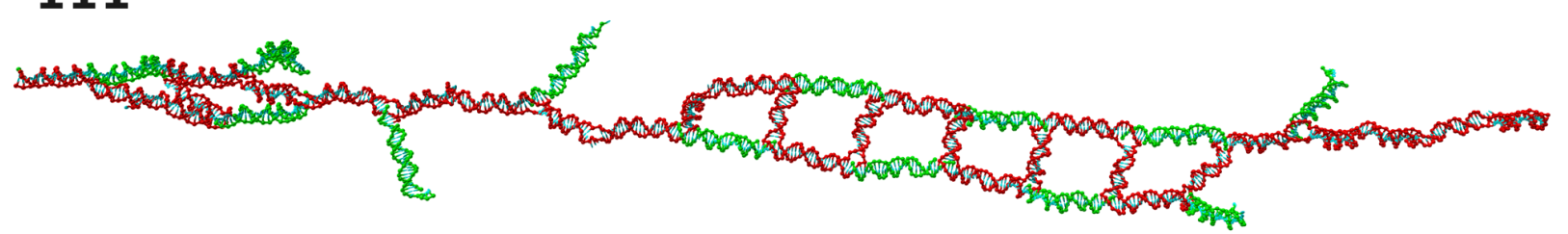

Figure S4. Example structures (intermediates in unfolding simulations) illustrating a linker detachment event for the extended motif along with the corresponding section of the force-extension curve. 


\section{WILEY-VCH}

\section{SUPPORTING INFORMATION}

1 S. Whitelam, E. H. Feng, M. F. Hagan, P. L. Geissler, Soft Matter, 2008, 5, 1251-1262

2. G. M. Torrie and J. P. Valleau, J. Comp. Phys. 1977, 23, 187-199

3. S. Kumar, J. M. Rosenberg, D. Bouzida, R. H. Swendsen and P. A. Kollman, J. Comp. Chem. 1992, 13, $1011-1021$.

4. J. Russo, P. Tartaglia and F. Sciortino, J. Chem. Phys. 2009, 131, 014504.

5. M.C. Engel, D. M. Smith, M.A. Jobst, M. Sajfutdinow, T. Liedl, F. Romano, L. Rovigatti, A.A. Louis and J.P.K. Doye, ACS Nano 2018, 12, 6734-6747. 